\title{
AN OVERVIEW OF ORACLE CHART BUILDER AND MAPVIEWER
}

\author{
Lory Molesky and Jayant Sharma \\ Oracle Corporation \\ lory.molesky@oracle.com, jayant.sharma@oracle.com
}

Abstract: Oracle Chart Builder is a real-time Java charting API. It enables more effective communication and analysis of information for business graphics and performance applications. MapViewer is a programmable tool for rendering maps using spatial data managed by Oracle Spatial. MapViewer provides tools that hide the complexity of spatial data queries and cartographic rendering, while providing customizable options for more advanced users. This paper presents an overview of data visualization features in the $9 i$ Application Server product from Oracle.

Key words: Charting, map display, Oracle 9i Application Server, visualization

\section{INTRODUCTION}

Web-based and other applications benefit from the ability to visualize data. For example, a brokerage firm may host a stock trading web site that displays charts for market data and fundamental data. A web hosting company may provide users with various charts displaying web statistics. A business intelligence application may display average household income for U.S. census blocks in a given region of the country.

However, enterprises face serious challenges when building applications that visualize data. These challenges include: 
- Interactive Visualization and Visualizing High-Bandwidth Feeds Interactive charting applications must provide real-time response, otherwise users will become impatient and go elsewhere. Highbandwidth data feeds-where thousands of charts must be updated frequently - such as real-time market data feeds or constantly changing system load conditions, require short batch cycle times.

- Displaying Time-Related Data

Displaying time-related charts is difficult, because data sets are often defined over numerous frequencies and contain different numbers of observations over time. Automating this task is important, because so much data is time-related.

- Integrating Map Display with Business Graphics and Reports Much business information has an implicit location component. Displaying it on a map, therefore, is an effective means of communicating this information. The viewer can also quickly grasp spatial relationships (such as proximity) between relevant business entities that would not be explicit in a tabular report or chart.

Chart Builder generates charts in a fraction of a second, and provides extensive support for time series data. One unique and powerful feature of Chart Builder is support for automatic time scaling, which maximizes the readability of the time axis. With Chart Builder, developers do not need to explicitly define time axis labels. This enables the generation of a wide range of charts without custom programming.

MapViewer generates thematic maps from data (spatial and non-spatial) and metadata stored in an Oracle database server. One form of thematic map allows the placement of charts (pie, bar, line, or area) on a map. For example, one can display quarterly sales by region, or gross sales for each major product category by store location.

This paper presents an overview of the above features that are part of the Oracle $9 i$ Application Server (9iAS). The remainder of this paper is structured as follows. Sections 2 and 3 describe Chart Builder, while MapViewer is presented in Sections 4 and 5. Section 6 give one example of integrating Chart Builder and MapViewer. Finally Section 7 summarizes the functionality and benefits of visualization components in $9 i \mathrm{AS}$.

\section{CHART BUILDER FEATURES}

Chart Builder supports numerous variations on all popular chart types. Chart Builder's intelligent default settings allow developers to easily create 
charts with just a few method calls. Developers can customize virtually any chart attribute.

\subsection{Chart Timeseries data}

Because the need to chart time series data is so common, Chart Builder provides extensive support in this area. Chart Builder automatically scales time axis labels to maximize the readability of the chart. Developers supply only the data set (in the form of date/value pairs). All axis labels, including the choice of labels for the time axis, are automatically derived by Chart Builder's internal algorithms.

For example, consider the case where the time axis is defined by daily dates. When only a few days (10) are provided, each day is labeled. Supplying more days (in this case 60) results in labeling full month names. The labeling of hours and minutes is also supported.
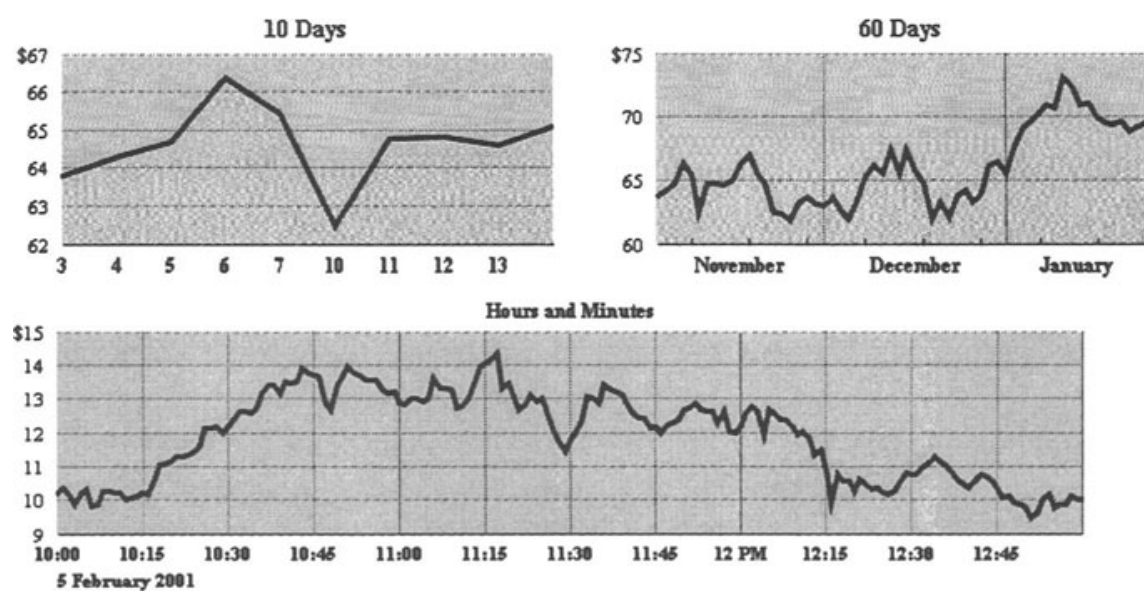

\subsection{Deploy charts in multiple languages}

Chart Builder generates axis labels using the language specified by the current Java Locale setting of the application. In addition, developers can customize the format of numerical axis labels by choosing from various numerical and currency formats.

\subsection{Generate Images in GIF, WBMP, and JPG Formats}

Chart Builder uses the Java BufferedImage class to create in-memory raster images. Developers can create chart images in GIF and WBMP formats by passing the buffered image to the standard encoding routines 
included with Chart Builder. GIF is the recommended file format for web sites, while the WBMP format is ideal for wireless devices. Developers can also encode charts using JPG encoders included with Java. However, this format is not recommended because it was designed for photographs.

\subsection{Create charts in under a second}

Chart Builder creates charts for typical data sets in a fraction of a second-including data sets containing hundreds of points. The following description refers to performance measurements of the time (in seconds) taken to create a single line chart containing either $10,100,500$, or 1000 points, in both cold and warm start scenarios. These performance measurements were taken on a modest laptop (300 MHz Pentium III, with 128 megabytes of memory, running Windows NT).

The cold start scenario measures the time taken to create a chart under the least favorable caching conditions-when charting methods are not cached in memory.

Under cold start conditions, it takes Chart Builder less than half a second to create the raster image of a line chart containing 100 points, and about three-quarters of a second to chart 1000 points.

Warm start performance measures the time taken to create charts after at least one chart has been created, as is the case when creating batches of charts. It takes Chart Builder about one fifth of a second and one half of a second to create an in-memory raster and generate the GIF file for 100 and 1000 points, respectively.

Applications that create more than one chart-where performance expectations follow the warm start scenario-include server-side applications used to create multiple charts images per request, or chart services that remain active between requests.

\section{CHART BUILDER DEPLOYMENT}

\section{DEPLOY INTERACTIVE CHARTS}

Chart Builder supports interactive charts in Java applications and applets, JavaServer Pages (JSP), and pure HTML contexts. Chart Builder provides mechanisms to detect events, such as mouse clicks, mouse entry, and mouse exit within a chart element (chart elements are individual pie slices, individual bars, etc.). Support for these methods is tailored to the runtime context (support for JSP environments is discussed in a separate section):

- Java Applications and Applets Contexts

Chart Builder provides access to the Java event model, enabling users to 
detect mouse events on individual chart elements. This model gives developers complete flexibility to associate actions with chart events.

- HTML Contexts

In an HTML context, interactive charts are provided by chart image maps. Chart image maps are automatically generated by Chart Builder, and associate mouse events on a chart with various actions. For example, entry into a bar can trigger a data drill down using image replacement, a link to a URL, or both. Both client-side and server-side image maps are supported.

The next example illustrates an interactive chart that could be implemented in either a Java application, a Java applet, or HTML. The
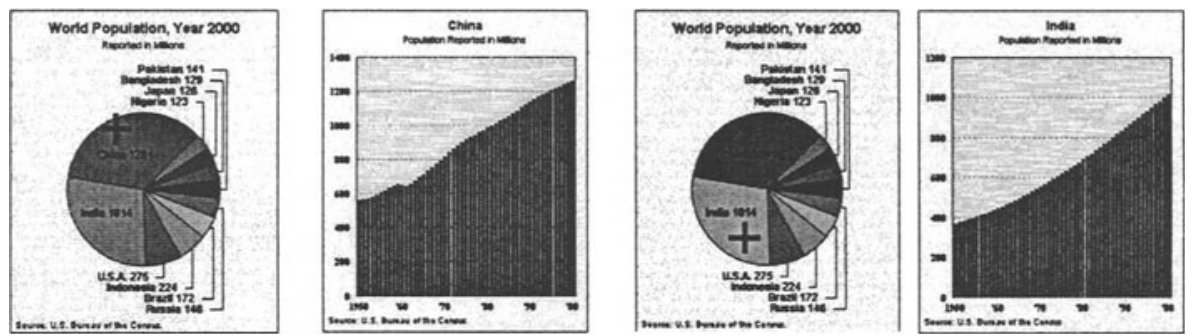

example detects cursor entry into any pie slice, highlights the slice, and displays a bar chart next to the pie chart that provides detail about the pie slice.

Placing the cursor in the slice labeled China highlights the China pie slice, and triggers the display of the China bar chart; if the cursor is moved to the slice labeled India, the India pie slice is highlighted, and the China bar chart is replaced by the India bar chart.

This technique, side-by-side images triggered by mouse overs, illustrates one of the many ways in which Chart Builder facillitates the development of effective user interfaces.

\section{DEPLOY CHARTS IN JSP ENVIRONMENTS}

JavaServer Page (JSP) technology is attractive because it allows enterprises to provide dynamic web content. Chart Builder's speed and image generation capabilities makes real-time response possible for JSP applications that embed charts. 


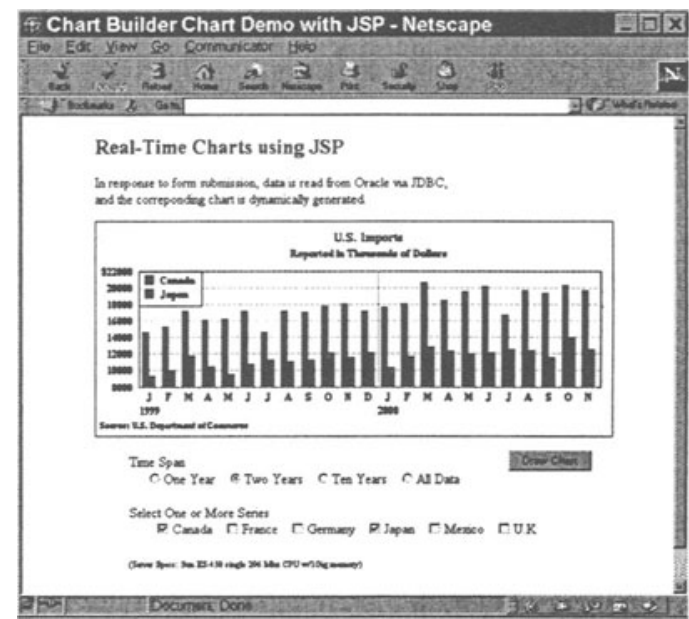

In summary Oracle Chart Builder provides a Java class library that lets you easily generate charts to display your data. It enables more effective communication and analysis of information for applications using charts. The following sections provide an overview of 9iAS MapViewer and its integration with Chart Builder.

\section{OVERVIEW OF ORACLE 9iAS MAPVIEWER}

The Oracle9i Application Server MapViewer (or simply, MapViewer) is a programmable tool for rendering maps using spatial data managed by Oracle Spatial. It can be deployed in a platform-independent manner and is designed to integrate with map-rendering applications.

MapViewer includes three main components:

- A rendering engine (Java class library) that provides cartographic rendering capabilities

- An XML API that provides a programmable interface to MapViewer

- A map definition tool that can be used to manage the metadata in a spatial database

The rendering engine connects to the Oracle database through JDBC. It also loads the map definitions, styling rules, and symbology from the database, and applies them to the retrieved spatial data. 
The XML API provides a convenient interface for high-level application developers, for submitting a MapRequest to the mid-tier MapViewer and handling its MapResponse. The map definition tool simplifies the process of creating and managing map, theme, and symbology information in the backend database.

The primary benefit of MapViewer is its integration with Oracle Spatial. It is not a full-featured web map or spatial application server.

\subsection{Basic Flow of Action}

With MapViewer, the basic flow of action involves two steps:

1. The client requests a map, passing in the map name, data source, center location, map size, and optionally other data to be plotted on top of a map.

2. The server returns the map image (or a URL for the image) and the minimum bounding rectangle (MBR) of the map.

\subsection{MapViewer Concepts}

When an application uses MapViewer, it applies specific styles (such as colors and patterns) to specific themes (that is, collections of spatial features, such as cities, rivers, and highways) to render a map (such as a GIF image for display on a Web page). For example, the application might display a map in which state parks appear in green and restaurants are marked by red star. A map typically has several themes representing political or physical entities, or both. For example, a map might show national and state boundaries, cities, mountain ranges, rivers, and historic sites. When the map is rendered, each theme represents a layer in the complete image.

\section{DEFINING STYLES, THEMES, AND MAPS}

MapViewer lets users define maps, styles, and themes, including the rules for applying one or more styles to each theme. These maps, styles, themes, and associated rules are stored in the database in map definition tables under the Oracle Spatial (MDSYS) system schema, and they are visible to users through metadata views. A set of basic styles is inserted automatically when MapViewer is installed. The set of styles, themes, and maps that users can access is determined by metadata views (USER_SDO_STYLES, USER_SDO_THEMES, and USER_SDO_MAPS) 
collectively referred to as a user's mapping profile. Users can manage styles, themes, and maps with the Map Definition Tool.

\subsection{Styles}

A style is a visual attribute that can be used to represent a spatial feature. The basic map symbols and legends for representing point, line, and area features are defined and stored as individual styles. Each style has a unique name and defines one or more graphical elements in an XML syntax. Each style is of one of the following types:

- Color: a color for the fill or the stroke (border), or both.

- Marker: a shape with a specified fill and stroke color, or an image.

- Line: a line style (width, color, end style, join style) and optionally a center line, edges, and hashmark. Lines are often used for linear features.

- Area: a color or texture, and optionally a stroke color. Areas are often used for polygonal features such as counties and census tracts.

- Text: a font specification (size and family) and optionally highlighting (bold, italic) and a foreground color.

- Advanced: a composite used primarily for thematic mapping..

\subsection{Themes}

A theme is a visual representation of a particular data layer. Each theme is associated with a specific spatial geometry layer, that is, with a column of type MDSYS.SDO_GEOMETRY in a table or view. For example, a theme named US_States might be associated with the STATE_SHAPE spatial geometry column in a STATES table.

All the themes for a database user are stored in that user's USER_SDO_THEMES view.

\subsubsection{Styling Rules}

Each theme is associated with one or more styling rules. The styling rules for each theme are expressed using an XML syntax, such as in Example 5.2.1 for an Airport theme.

Example 5.2.1 XML Definition of Styling Rules for an Airport Theme

<?xml version="1.0" standalone="yes"?> 


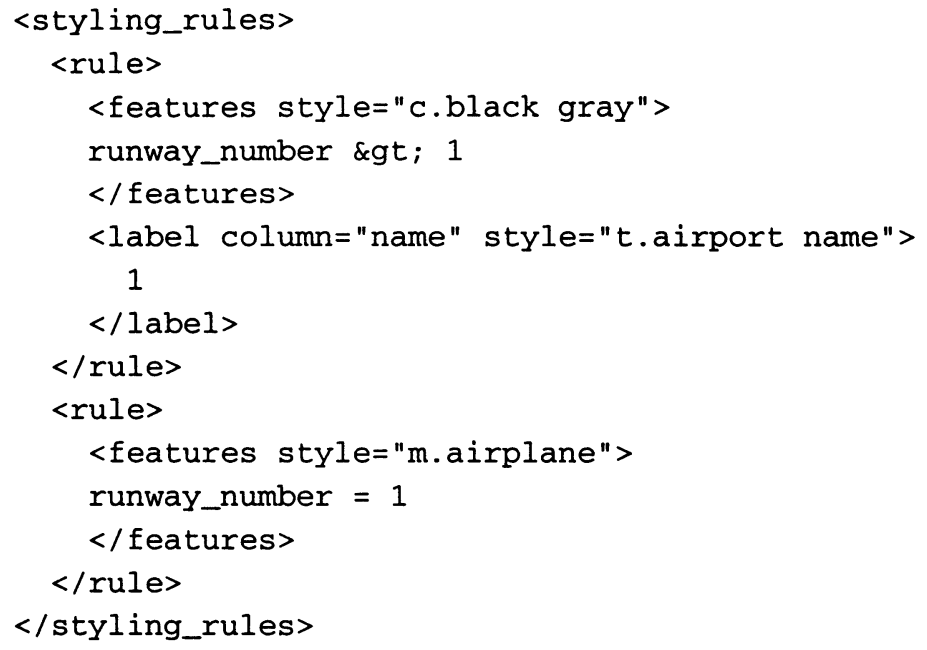

Each styling rule has a required <features> element and an optional $<$ label>element. The <features> element specifies which rows in the base table or view will be selected based on its attribute value, and the style to be used for those selected features. The <label> element specifies whether or not to annotate the selected feature, and if so, which column in the base table or view to use for text labels.

In Example 5.2.1, there are two styling rules associated with the Airport theme:

- The first rule specifies that only those rows (features) that satisfy the condition runway_number \&gt; 1 (that is, runway number greater then 1) will be selected, and these will be rendered using the style named c.black gray. Any valid SQL WHERE clause conditions can be used as value of the <features> element, which provides flexibility in labeling and annotation. If no value is supplied, no WHERE clause condition is applied. For example, if the definition had been the following (that is, omitting the runway_number \&gt; 1 condition):

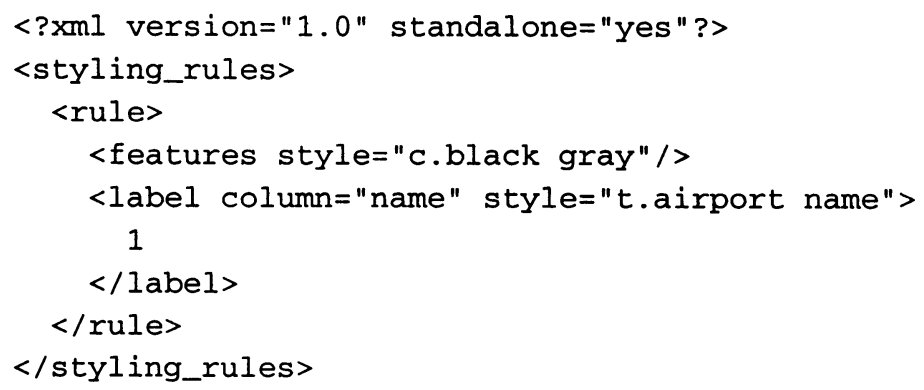


then all airport features would be selected and would be rendered using the Color style named c.black gray.

The first rule also has a <label> element, which specifies that the NAME column in the base table or view will be used to annotate each airport using the text style t.airport name. The value of the label element is used to determine whether the feature will be annotated or not. If the value is greater than zero, the feature will be annotated. In this case, because the value is 1 , all the features specified by the <features> element will be annotated, using the values in the NAME column. If the value is less than or equal to zero, that feature will not be annotated.

- The second rule, which applies to those airports with only one runway, does not have a <label> element, thus preventing all such airports from being annotated. In addition, the features that satisfy the second rule will be rendered using a different style as specified in its $<$ features $>$ element.

\subsubsection{Thematic Mapping}

Thematic mapping refers to the drawing of spatial features based on their attribute values. For example, a Counties theme can be drawn using colors with different hues that map directly with the population density of each county.

To achieve thematic mapping, specify Advanced styles in the styling rules associated with a theme. You must specify attribute columns in the base table or view whose values will be used to determine exactly how a feature will be rendered thematically. Example 5.2.2 is the XML definition for an Earthquakes theme.

Example 2-2 XML Definition of Styling Rules for an Earthquakes Theme

<? xml version="1.0" standalone="yes"?>

<styling_rules theme_type="nature" >

<rule column="RICHTER_SCALE" >

<features style="v.earthquakes" >

$</$ features $>$

$</$ rule $>$

$</$ styling_rules $>$

Note that the theme in Example 5.2.2 has only one rule. The <rule> element includes an attribute named column that does not appear in the Airport theme in Example 5.2.1. The column attribute specifies one or more columns (comma-separated) that provide the attribute values needed for 
thematic mapping. The style specified for the features element is named v.earthquakes, and it is an Advanced style.

Another part of this definition of the Earthquakes theme referred to in Example 5.2.2 specifies the base table that contains the data to be rendered. This table must contain a column named RICHTER_SCALE in addition to a column (of type MDSYS.SDO_GEOMETRY) for the spatial data. The RICHTER_SCALE column must be of type NUMBER. To understand why, look at the definition of the Advanced style definition in Example 5.2.3.

Example 5.2.3 Advanced Style Definition for Earthquakes Theme

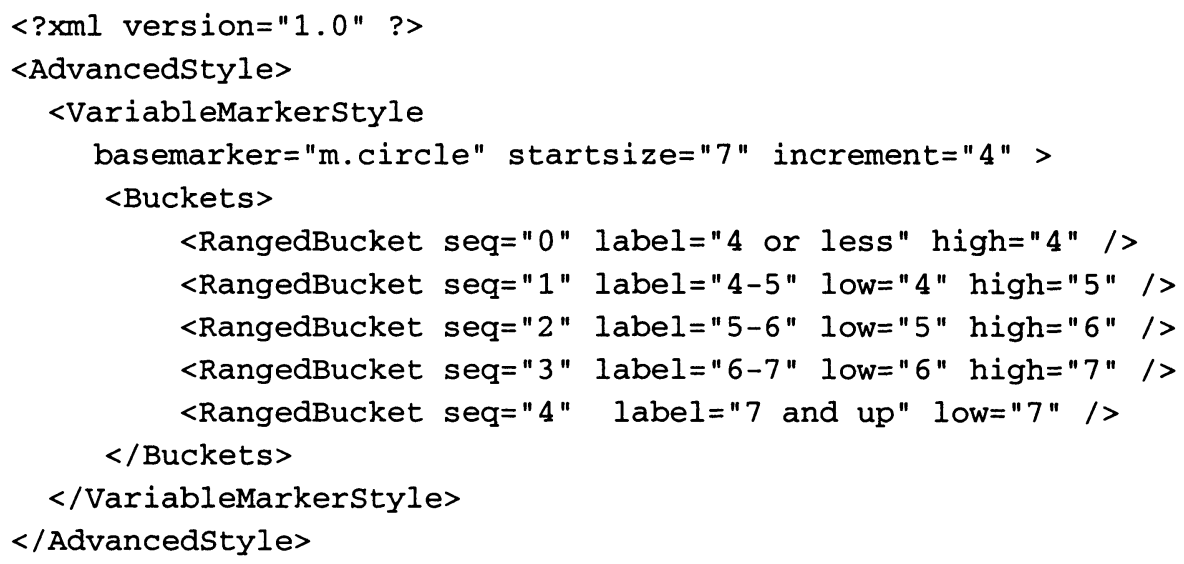

This style specifies that the marker named m.circle is used to indicate the location of an earthquake. The size of the marker depends on the numeric value of the RICHTER_SCALE column for that row, as specified by the $<$ Buckets $>$... < B Buckets $>$ tag. In this example there are five buckets, each covering a predetermined range of values. For example, if an earthquake is of magnitude 5.7 on the Richter scale, the marker size will be 15 pixels $(7+$ $4+4)$, because the value 5.7 falls in the third bucket $(5-6)$ and the starting marker size is 7 pixels (startsize $=" 7 ")$ with an increment of 4 for each range (increment="4").

\subsection{Maps}

Maps are collections of themes with their scale ranges. A map is a stack of themes rendered on top of each other. A map also has an associated coordinate system that all themes in the map must share. For example, if the map coordinate system is 8307 (a Spatial Reference System Identifier defined by Oracle Spatial which indentifies Longitude / Latitude (WGS 84), a common system used for GPS devices), all layers in the map must have geometries defined using that coordinate system. 
Maps are created by specifying a base map name or by using the programming interface to add themes, and then rendering the map into a window. The order in which the themes are added determines the order in which they are rendered, with the last specified theme on top, so be sure you know which themes you want in the background and foreground.

All base map names and definitions for a database user are stored in that user's USER_SDO_MAPS view. The DEFINITION column in the USER_SDO_MAPS view contains an XML description of a map. Example 5.3.1 shows a map definition.

Example 5.3.1 Map Definition

<? xml version="1.0" ?>

$<$ map_definition>

<theme name="theme_us_states" min_scale="10" max_scale="0" />

<theme name $="$ theme_us_parks" min_scale $=" 5 "$ max_scale $=" 0 "$ />

<theme name="theme_us_highways" min_scale="5" max_scale="0" />

$<$ theme name $=$ "theme_us_streets" min_scale $=0.05 "$ max_scale $=0 " 0$ "

1>

$</$ map_definiton $>$

A map is made up of themes, each of which has a scale range within which it is displayed. Referring to Example 5.3.1, the theme named theme_us_streets is not displayed unless the map request is for a level of detail within the specified scale range.

The display order of themes is the same as their order in the map definition. In Example 5.3.1, the theme_us_states theme is rendered first, then theme_us_parks, then theme_us_highways, and finally (if the current scale is within its specified range) theme_us_streets.

\subsection{Displaying Charts on Maps}

Displaying charts at particular location on a background requires associating a chart type with a set of point geometries. A subtype of the Advanced Style defines the chart type and presentation. A corresponding theme associates the location with the data required for generating charts at those locations.

An example of an Advanced Style for defining a Pie Chart is:

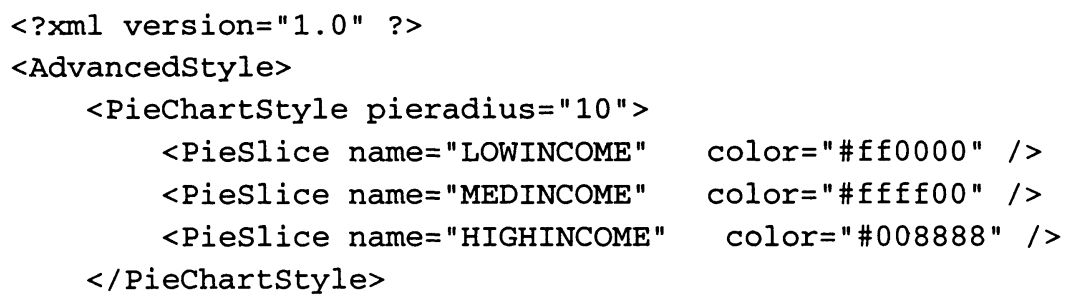


$</$ AdvancedStyle $>$

while the corresponding theme definition is:

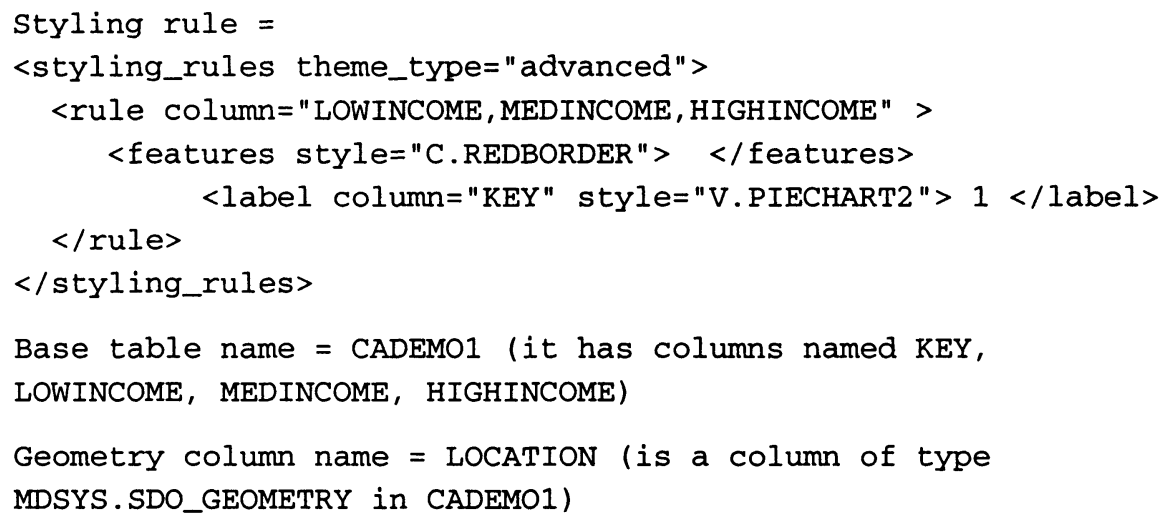

The following figure shows a sample map generated when using the above theme in its definition. 


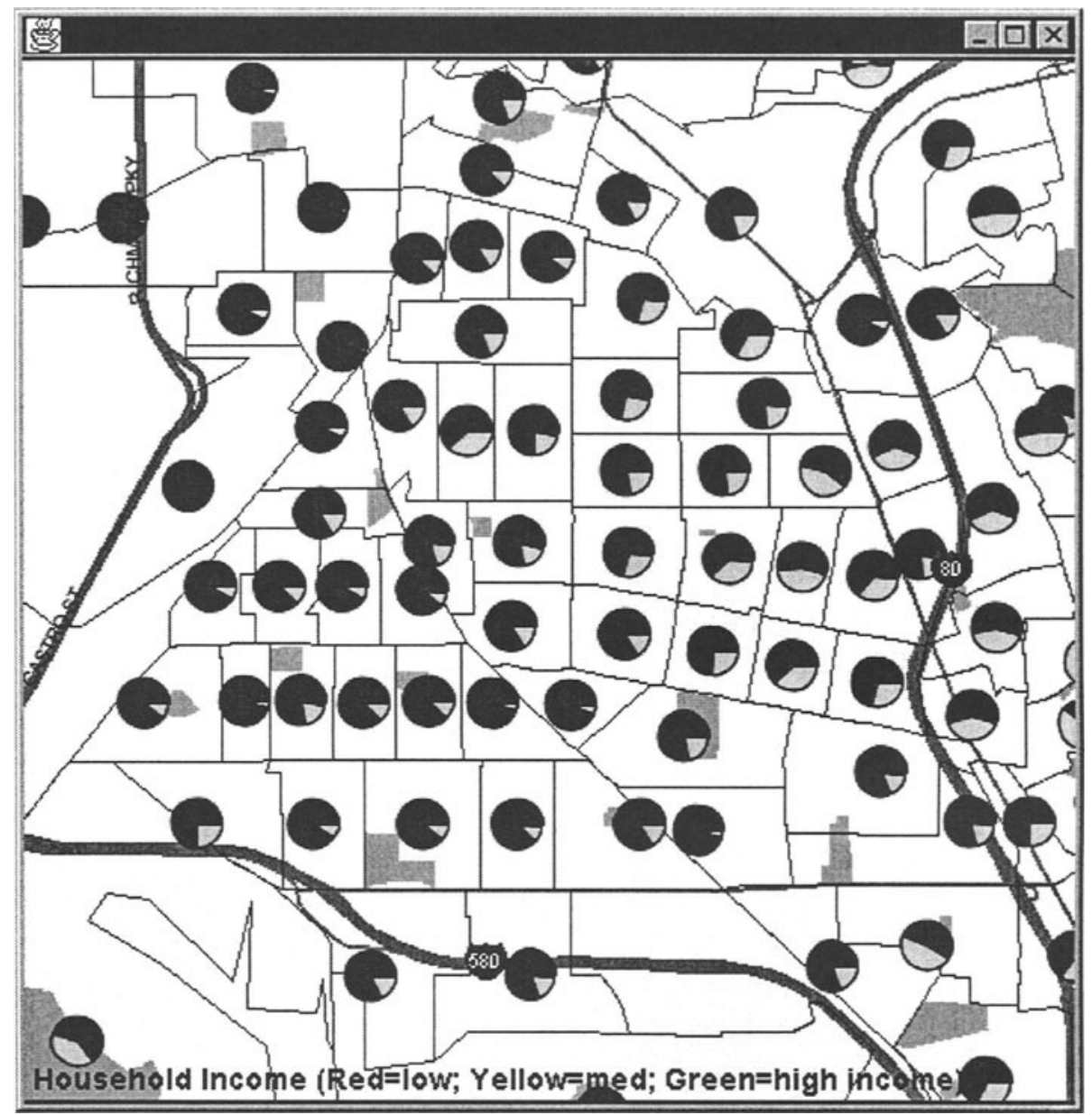

Figure 5.1: Sample map showing use of Chart Builder with MapViewer.

\section{CONCLUSIONS}

Oracle Chart Builder and MapViewer provide a Java class library and servlet that let you easily generate charts and maps to display your data. They enable more effective communication and analysis of information for applications using charts and maps. Their primary benefit is tight integration with the Oracle internet platform, that is, the $9 i$ data and application servers and their application development and deployment infrastructure. 